\title{
Continuity of care for people with multimorbidity: the development of a model for a nurse-led care coordination service
}

\author{
AUTHORS \\ KATE M DAVIS RN MCISC ${ }^{1}$ \\ MARION C ECKERT RN DNsg ${ }^{1}$ \\ AMANDA HUTCHINSON PhD ${ }^{2}$ \\ JOANNE HARMON $\mathrm{PhD}^{3}$ \\ GREG SHARPLIN MPsych ${ }^{1}$ \\ SEPEHR SHAKIB MBBS, $\mathrm{PhD}^{4,5}$ \\ GILLIAN E CAUGHEY PhD ${ }^{5,6,7}$

\section{CORRESPONDING AUTHOR} \\ KATE M DAVIS Rosemary Bryant AO Research \\ Centre, University of South Australia \\ City East Campus, Playford Building P4-27, \\ North Terrace, Adelaide SA 5000. \\ Phone: + 61883022129. \\ Email: kathryn.davis@mymail.unisa.edu.au
}

1 Rosemary Bryant AO Research Centre, UniSA Clinical \& Health Sciences, University of South Australia, Adelaide, Australia.

2 UniSA Justice \& Society, Social Work and Social Policy, University of South Australia, Adelaide, Australia.

3 UniSA Clinical \& Health Sciences, University of South Australia, Adelaide, Australia.

4 Clinical Pharmacology, Royal Adelaide Hospital, Adelaide, Australia.

5 Discipline of Pharmacology, Adelaide Medical School, University of Adelaide, Royal Adelaide Hospital, Adelaide, Australia.

6 Registry of Senior Australians, South Australian Health and Medical Research Institute, Adelaide, Australia.

7 UniSA Clinical \& Health sciences, University of South Australia, Adelaide, Australia.

\section{ABSTRACT \\ Objective: To collaboratively develop a model of nurse-led care, within a multidisciplinary team and support continuity of care at the primary- secondary interface for people with multimorbidity.}

Background: Existing models of care are frequently based on a medical model, designed to manage a single disease condition, and thus pose a significant challenge to provide healthcare for people with multimorbidity. Particular design elements for models of care affecting the primary-secondary interface have been previously demonstrated, however, these have not been applied to the development of a nurse-led model of care for people with multimorbidity.
Study design and methods: This paper, the first in a series of two, is part of a broader action research study and reports on the development of a model which will subsequently be assessed in terms of feasibility to provide a nurse-led care coordination service for people with multi-morbidity. This paper reports on the first action research cycle and methodology including a literature search, stakeholder engagement forums, validation workshop, team meetings, and professional engagement and validation.

Results: Data from two stakeholder forums were sorted into 257 'structure, process and outcome' statements and 86 goal related statements.

These were cross referenced with design elements on models of care from the literature and finally aggregated into themes. The aggregated themes 
were then integrated into a model of care for a nurse-led care coordination service. The model consists of an overarching component, 4 domains and 6 operational areas with underpinning criteria.

Conclusion: Through stakeholder consultation, consideration of the strengths of previous models and building blocks, a new nurse-led model of care that provides a pathway for transitional healthcare management at the primary-secondary interface has been developed. Inclusion of governance and culture within the model's domains enables the approach to be pragmatic and adaptable, contributing to the potential for successful change management and model implementation in the clinical workplace. Further evaluation and refinement of the model is planned and will be reported on, in part 2 of this two-part series.

Implications for research, policy, and practice: These findings provide direction for model implementation and further research required regarding nurse-led models of care. The supporting documents, systems, and processes reported, positions the model to support change and guide clinical practitioners and nursing management working at the primary-secondary healthcare interface. The future success of model implementation could provide evidence for health workforce policy and coordinated healthcare management.
What is already known about this topic:

- Particular design elements for models of care affecting the primary-secondary interface have been previously demonstrated.

- Interventions delivered at the primary-secondary care interface, particularly stepped care and models of shared care are effective for the management of depression.

- Established model design elements and interventions to improve continuity of care at the primary-secondary interface have not been applied to nurse-led models of care for chronic conditions and still require development within research settings.

\section{What this paper adds:}

- A new person-centred nurse-led model of care coordination, with healthcare management activities intended to support and enable development of the person's agency in their healthcare optimisation.

- A model with specific domains and criteria with the potential for application to nurse-led services across primary and secondary settings, for a range of patients.

- Inclusion of governance and culture as domains within the model, to enable the best possibility for change, model implementation and continuity of care between the primary-secondary healthcare interface.

Key words: nurses, 'model of care', 'continuity of patient care', 'chronic disease management', 'transition and care'

\section{INTRODUCTION}

This article, the first in a series of two, is part of a broader action research study to evaluate the feasibility of a nurseled care coordination service to improve continuity of care for people with multimorbidity. ${ }^{1}$ This paper reports on the development of a model for nurse-led care coordination using stakeholder consultation and validation processes as part of the first action research cycle within a broader study. The second paper in the series reports on an implementation study and evaluates the model as part of subsequent action research cycles. Multiple design elements have previously been identified for models of care both within Australasia and internationally to support care at the primary-secondary interface. ${ }^{2,3}$ These elements provide direction for services to aim for continuity of care; however, a medical approach underpins the focus within the elements, and it is therefore timely to explore nurse-led healthcare models at the primary- secondary interface (primary health care-community/ general practice and secondary care-acute/tertiary care, outpatient setting).

Continuity of care is recognised as essential to high quality healthcare and relates to an individual's experience of connection, coherence and consistency of care delivered over time. ${ }^{4,5,6}$ Continuity of care is relevant across a range of clinical settings, and has different components: informational, management, and relational, which can all be measured by particular criteria within continuity of care measurement indices. ${ }^{7}$ The emphasis on each component of continuity differs depending on the type and setting of care. ${ }^{6}$ Despite an understanding of continuity of care, ${ }^{6}$ it is evident that providing and promoting continuity of care for people with multimorbidity is challenging within current healthcare systems and models of care. ${ }^{8}$ 
Multimorbidity is commonly defined as the co-existence of two or more chronic health conditions in an individual. ${ }^{9}$ Increasing prevalence and complexity of multimorbidity within general populations is a global phenomenon and more coordinated models of healthcare delivery, ones that facilitate continuity of care for people with multimorbidity, are required. ${ }^{8,10-24} \mathrm{~A}$ person-centred, nurse-led approach, rather than a single-disease management program could provide more effective, high-quality care..$^{25}$

\section{BACKGROUND}

A healthcare delivery model is underpinned by a theoretical framework that guides the practices and interventions of patient assignment and care which support the underlying philosophy of care. ${ }^{26}$ Existing models of care are frequently based on a medical model, designed to manage a single disease condition, and thus pose a significant challenge when considering people with multimorbidity. ${ }^{24}$ Due to the complexity of care requirements generated from chronic and multiple disease situations, these people require continuity of care across the health sector. When achieved, this is correlated with both improved patient outcomes and satisfaction. ${ }^{5}$ A single disease-focused model of care is unsuitable for managing, measuring and improving the health of people with multimorbidity. ${ }^{24}$ The literature calls for a move to a patient/person-centred model of care, one that is holistic and able to facilitate coordination and effective management of the multiple complex health and psychosocial needs of the individual person. ${ }^{27-29}$

The chronic care model (CCM) set a precedent to systematically and holistically manage care for people living with chronic health conditions, ${ }^{27}$ and provided a foundation for the development of subsequent models that were more consumer focused than traditional medical models. Consumer focused models included: chronic disease integrated care, ${ }^{28}$ guided care, ${ }^{29}$ partnership, ${ }^{26}$ case management, ${ }^{30,31}$ transitional care, ${ }^{32}$ person-centred care and patient-centred care models.33,34

The term 'patient-centred care' refers to a model of care that is "respectful of and responsive to individual patient preferences, needs, and values, and ensuring that patient values guide all clinical decisions". 35 The literature tracks the evolution of the patient-centred care model and notes the transition to the term person-centred.33,34,36-42

This move in terminology from patient-centred to personcentred care delivery aims to acknowledge the entirety of a person's needs and preferences, beyond only the clinical or medical. 33 The concept of a person driving their own healthcare decisions is now recognised and considered the gold standard in health care. 33 Two key elements of person-centred care include identification of a lead care coordinator and integrated communication. The former enables one person to be the primary contact for the person, their healthcare team and all service providers to facilitate communication and transitions across settings. ${ }^{33}$ The latter requires establishing a system that supports simultaneous access to care plans across settings. Person-centred care has been shown to "advance concordance between care provider and patient on treatment plans, improve health outcomes and increase patient satisfaction". ${ }^{6}$

The transitional care model (TCM) provides and facilitates time-limited services during episodes of acute illness across settings, and has the potential to be adapted to a range of services. ${ }^{2}$ This evidence-based model of care includes a focus on person-centredness, the use of advanced practice registered nurses, care coordination, continuity of care and the use of evidence-based tools and interventions. However, the model does not overtly address organisational culture or governance - two key areas that can pose significant challenges to implementing new services.

A model of care inclusive of positive organisational culture strategies to promote good outcomes between primary and secondary healthcare sector transitions is required. Organisational culture embodies our collective ways of thinking, feeling and behaving in healthcare organisations and is acknowledged as a complex construct with many layers across health sectors. ${ }^{43}$ Braithwaite's systematic review on organisational and workplace cultures and patient outcomes found there was a "consistently positive association held between culture and outcomes across multiple studies, settings and countries", 44 supporting consideration of this concept within a new model of care.

Effective governance at the primary-secondary interface is also critical for continuity of care. Successful governance mechanisms are characterised by clear leadership, built trust, articulation of organizational goals and consideration of the workforce through planning, implementation, and evaluation of change. 45 Nicholson's systematic review of governance models for primary and secondary care provides detail on the necessary elements for effective governance, ${ }^{46}$ thus providing sound direction for the application of governance elements to primary-secondary health services. Yet the elements are not positioned within an overall recommended healthcare or nurse-led model. A disruptive innovation is required, ${ }^{47}$ a nurse-led care coordination service, specifically designed to influence outcomes at the primary-secondary interface.

The advent of the COVID-19 virus pandemic necessitates the examination of nurse-led models of care with a new sense of urgency. ${ }^{48}$ The drastic resource implications of COVID-19 on our existing health system mean there has never been a more suitable time to enact a new way of thinking. Keeping those at greatest risk out of hospital, yet with access to specialist services, whilst still engaged with primary care is imperative. The capacity to manage a traditional approach at the primary-secondary interface is evaporating and change 
is required. A nurse-led care coordination service could be a disruptive innovation in healthcare delivery that facilitates keeping people safe in the community.47,49 Nam's blog on disruptors in healthcare notes 'disruptive innovations cost less, and over time, do more'. ${ }^{47}$ An innovative and adaptive model of care which includes evidence-based design elements applicable across healthcare sectors is required. ${ }^{46}$ The critical element to this disruptive innovation in the current climate is to progress proactively, rather than ad-hoc, particularly when evidence-informed strategy is currently limited.

There is a paucity in nurse-led services providing continuity of care and a person-centred approach for people with multimorbidity. Furthermore, there is no guiding model of care that addresses the significant issues of healthcare culture and governance between the primary and secondary healthcare sectors. Regarding patient needs within a healthcare system, the literature notes that patients' four most pressing requirements are:

1 convenient access to providers (via telephone, internet or in person),

2 clear communication of individualised care plans,

3 support from a single coordinator of care who can help prioritise competing demands and continuity of relationships,

4 providers who listen to and acknowledge patients' needs, appreciate that these needs are unique and fluctuating, and have a caring attitude. 49

These requirements, clearly articulated by healthcare consumers, can be addressed when contextualised within appropriate organisational cultures, and governed effectively. The development of a nurse-led model of care applied within a multidisciplinary framework, underpinned by a person-centred approach, that addresses continuity of care issues, will seek to address the current problems of care fragmentation for people with multimorbidity, and respond to their most pressing needs.

\section{STUDY DESIGN AND METHODS}

\section{AIM}

The aim of the study was to collaboratively develop a model of care for a nurse-led care coordination service. A range of stakeholders were engaged, with a view to subsequently implement a pragmatic nurse-led model of care within a multidisciplinary team in an outpatient setting that could support continuity of care at the primary-secondary interface, for people with multimorbidity.

\section{DESIGN}

This paper is part of a broader action research study and reports on the development of a model which will subsequently be assessed in terms of feasibility to provide a nurse-led care coordination service for people with multi-morbidity. As part of the broader study, Kemmis and McTaggart's action research approach was used including: planning, action, reflection, observation, and revision planning. ${ }^{50}$

The broader study has 2 phases, ${ }^{1}$ the first phase and first action research cycle focused on model development and included a systematic review (PROSPERO registration number: CRD42018095780, submitted), stakeholder engagement forums, validation workshop and clinical team meetings. Reporting on the stakeholder engagement process aimed to capture stakeholder knowledge and experience by combining pragmatism and idealism into a workable model that addressed patient care delivered across the secondary and primary health care sectors. ${ }^{1}$ Phase 2 (to be reported in a subsequent paper) included further action research cycles, with a mixed methods approach and multiple data collection points with validated data collection tools. ${ }^{1}$ Phase 2 focused firstly on operationalising the model with implementation of service protocols, procedures, clinical guidelines, and the lead nurse care coordinator role, and secondly on evaluation of the nurses', multidisciplinary staff and patients' experiences of the nurse-led care coordination service and model.

\section{SETTING AND PARTICIPANTS}

Participants for this study included 44 stakeholders who attended one of two forums. Stakeholders included nurses, medical staff, an occupational therapist and pharmacists from within the outpatient clinic - multidisciplinary ambulatory consulting service (MACS); consumer advocates; 2 Aboriginal women who expressed representation for both the Aboriginal and the Torres Strait Island peoples' voices; healthcare executives from the primary and secondary healthcare sectors; general practitioners; practice nurses; nurse managers; academics and registered nurses. Eight stakeholders attended a follow up validation workshop.

\section{ETHICAL APPROVAL}

Ethics approval was obtained by the Human Research Ethics Committee (HREC) (reference number: HREC/17/RAH/552) at the University of South Australia (application ID: 200958) and the Central Adelaide Local Health Network (CALHN) (reference number: R20171204).

\section{DATA COLLECTION}

Data were primarily collected through a consultative process in two stakeholder forums and a validation workshop. Consultation data were further refined through meetings and professional discussions with the MACS team who were to implement the model. Data were also validated through a literature search. 


\section{Stakeholder forum}

Two stakeholder forums were facilitated by consumers, academics and healthcare professionals, each of 3 hours duration. On commencement of each forum a presentation was delivered to provide the context and aims of the forum. Four tables each with 5 to 6 participants worked through 5 activities related to the strategy, structure, process and roles required to implement a nurse-led service for people with multimorbidity. A final activity required participants to identify their top 3 goals for a nurse-led service to achieve success. A scribe for each table collected participant responses. The stakeholder forums resulted in a draft nurse-led model of care with an overarching component, 4 domains, 6 operational areas and underpinning criteria.

\section{Validation workshop}

The validation workshop was of three hours duration and facilitated by an academic and a health professional. The draft model along with background context and aims for the validation workshop were presented. Participants reviewed the domains and model criteria in table groups, with activities guided by facilitators. Two tables of approximately 4 people each reviewed the model, cognisant of its operational application in a pragmatic outpatient setting. The draft model data were then compared with 'Models of care' literature and a further iteration resulted. To ensure continuity of care for patients transitioning between the secondary and primary healthcare settings, the consequential model domains were then mapped to the Australian Primary Healthcare Nurses Association (APNA) 'building blocks for nurse-led clinics. ${ }^{51}$

\section{DATA ANALYSIS}

The broader study used recognised and validated instruments to collect data in relation to continuity of care, patient-centredness, workplace culture and the nurses' practice role. ${ }^{1}$ A concurrent approach to data collection using quantitative and qualitative methods within cycles allowed both sets of data to be interpreted together, providing a richer and more comprehensive response to research questions. ${ }^{1,52}$ This study, (Phase 1) focusing on stakeholder consultation and validation, adapted Braun and Clarke's methodology of thematic analysis, 53 to categorise and synthesise data into a workable model that could later guide the implementation of the nurse-led service. Finally, the consultation data and model domains were aligned with literature evidence and preliminary results of the systematic review (PROSPERO registration number: CRD42018095780, submitted). Synthesis of the outcomes from the stakeholder consultation process with the literature search and systematic review were key elements of the analytic process.

\section{RESULTS}

\section{STAKEHOLDER WORKSHOPS AND VALIDATION FORUM}

Data from the two stakeholder forums were sorted into 257 'structure, process and outcome' statements and 86 goal related statements, ${ }^{54}$ and finally aggregated into themes (see Tables 1 and 2). The aggregated themes were again presented in a draft model with an overarching component, 4 domains, 6 operational areas and underpinning criteria. Subsequently, information from the participant validation workshop was integrated into the draft model.

\section{TABLE 1: DEVELOPING THE MODEL FROM PARTICIPANT 'STRUCTURE, PROCESS AND OUTCOME' ACTIVITY:54 STATEMENTS (INCLUDING NUMBER OF RELEVANT STATEMENTS)}

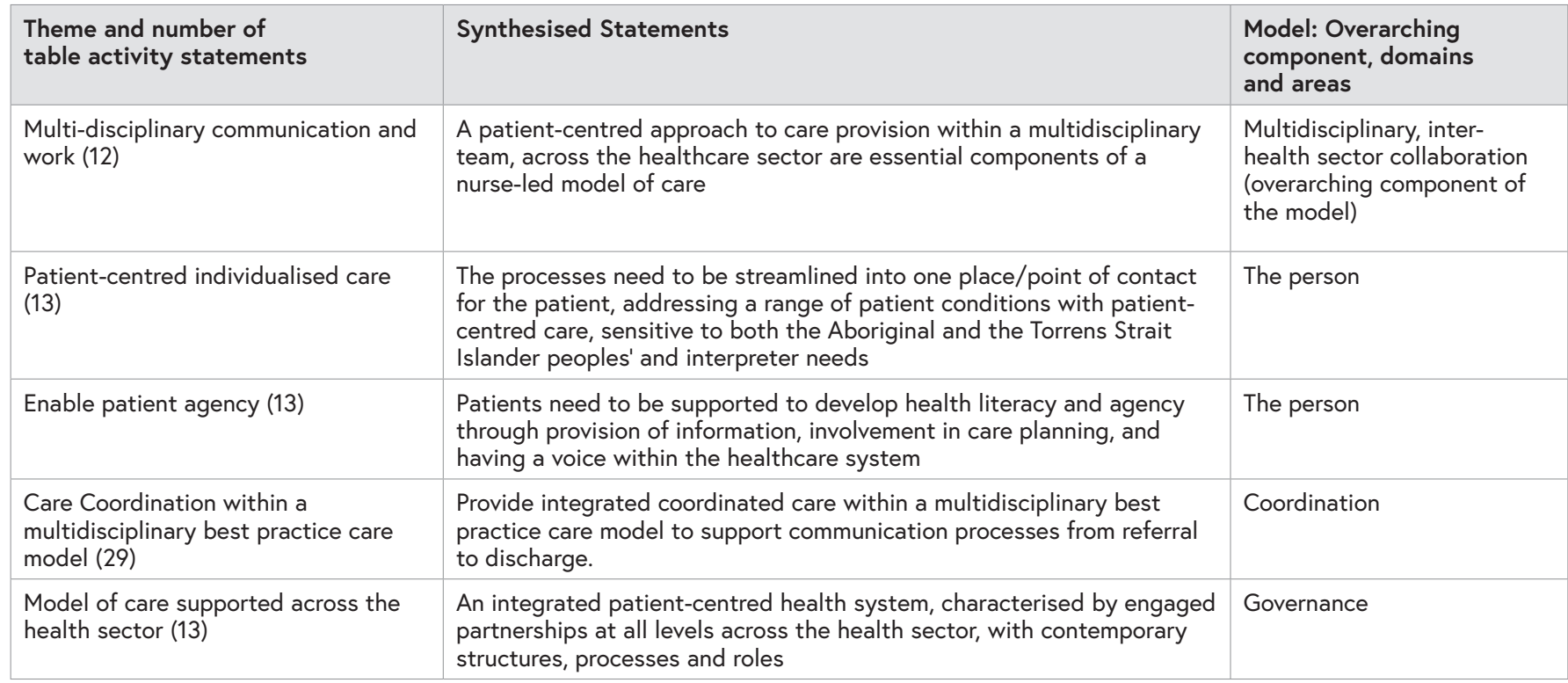




\section{TABLE 1: DEVELOPING THE MODEL FROM PARTICIPANT 'STRUCTURE, PROCESS AND OUTCOME' ACTIVITY: ${ }^{54}$ STATEMENTS (INCLUDING NUMBER OF RELEVANT STATEMENTS) (continued)}

Theme and number of
table activity statements
Disparate funding mechanisms (6)
Essential components of a valuable
model of care (9)
Communication at all levels within the
health system (18)
A culture that enables power balance
and good relationships across the
health sector (10)

Patient-centred care processes within a multidisciplinary team (26)

Patient and carer relationships (7)

Key performance Indicators and best practice (18)

Evaluation and improvement are multi-faceted (12)

Streamlined and congruent systems between the primary and secondary healthcare sectors (32)

Information technology (22)

Workforce development (10)

Statements subtotal $=257$

\section{Synthesised Statements}

Separate state and commonwealth funding models do not support continuity of care

A model of care and systems that involve a multi-disciplinary team, develops quality care plans and is integrated across the acute \& primary care sectors

Effective communication and coordination of care require appropriate transition management, good workforce relationships and streamlined health records

The healthcare culture requires balance between nursing and medical leadership with sharing of expertise and an understanding of scope of practice to empower nurses in nurse-led clinic roles

Nursing assessment and consultations need to be characterised by systematic assessment, conducted by educated and skilled nurses and supported by resources, tools, and software.

Appropriate referral, assessment, communication, goal setting, health coaching, self-monitoring, and care optimisation are required as part of the health management process. Care planning and interventions need to be supported by resources, tools, and software. The care process is linked with multi-disciplinary engagement, effective monitoring, and evaluation of care and key performance indicators.

Relationships and support are central to enable patients' agency in their healthcare management

Measurement of key performance indicators within the multidisciplinary team is a valued part of best practice and processes

Evaluation and improvement processes within the multidisciplinary team are an integral part of best practice

Systems, processes, and resources that support multimorbidity best practice guidelines, are patient-centred, enable patient flow, and support timely service accessibility are required

Disparate information technology and data management systems do not support effective communication or workflow

Skilled staff supported by systems that provide access to continuing professional development and education pathways
Model: Overarching component, domains and areas

Governance

Governance

Communication

Culture

Health assessment

Care processes

Patient, significant others and carer relationships

Clinical best practice

Evaluation and improvement

Systems, processes and resources

Systems, processes and resources

Systems, processes and resources

\section{TABLE 2: INTEGRATION OF PARTICIPANT GOAL STATEMENTS INTO THE MODEL}

Theme and number of goal statements

Communication and collaboration (13)

Patient/person-centred care (27)

Care coordination for people with multimorbidity (22)

Systematic process (7)

Cultural awareness (7)

Role (4)

Service characteristics (6)

Sub-total: goal statements

Total Statements

\section{Synthesised Statements}

Communication and collaboration with all providers and services across the healthcare sector and with the patient

Partnership with the patient in quality care that empowers and enables decision-making and involvement

Coordinated model of care that optimises health for people and ensures patient-centred, planned, managed and effective care

A systematic person-centred process of care

Culturally competent, skilled (advance practice) nurse-led services

Workplace and nursing role satisfaction along with advocacy and equity are underpinning values

The service needs to be sustainable, nimble and value learning
Model domain/area

Communication

The person

Coordination

Systems, processes and resources

The person

Systems, processes and resources

Systems, processes and resources 


\section{MODEL FOR A NURSE-LED CARE COORDINATION SERVICE WITHIN A MULTI-DISCIPLINARY CLINIC}

\section{Model Aims}

The key aim of the model (see Figure 1) developed by the authors is to provide a 'map' or guide for implementing and working in a nurse-led care coordination service within a multidisciplinary team. The model is intended to be implemented from the outpatient setting and enable continuity of healthcare for people with multimorbidity. Additionally, the model aims to facilitate well-co-ordinated transitional care between the secondary and primary healthcare settings; monitoring and keeping patients managed well in the community and reducing avoidable hospital admissions.

\section{FIGURE 1: MODEL OF CARE: NURSE-LED CARE COORDINATION SERVICE}

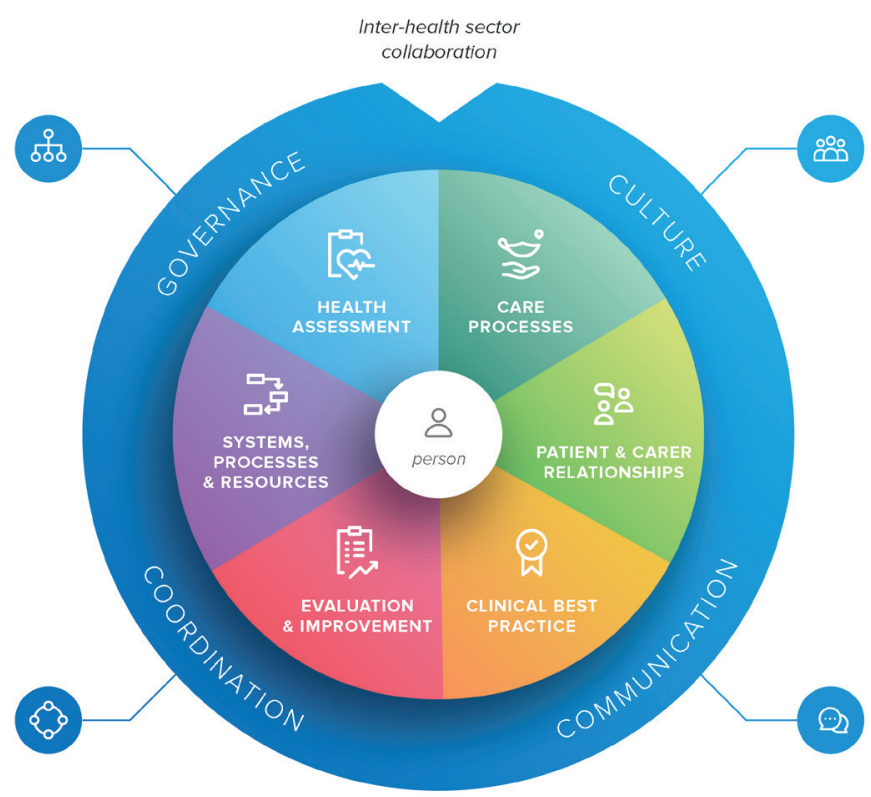

The Model: Overarching component, domains and operational areas

The model contains an overarching component, 4 domains, 6 operational areas and the person at the centre of the model. The overarching component of Multidisciplinary inter-health sector collaboration guides and provides cohesion for the domains and operational areas. A multidisciplinary approach to care for people with multimorbidity is supported in the literature and was promoted and validated as an essential component at the stakeholder forums..$^{56,57}$ Similarly, inter-health sector collaboration supports health teams to communicate consistently and effectively between the secondary and primary healthcare sectors, to achieve continuity of care.55

\section{Inner circle, the person}

This represents the person/patient at the centre of the nurseled care coordination service. All healthcare management is intended to enable support and development of the person's agency in their healthcare optimisation.

\section{Blue outer circle and inner coloured triangles}

The outer circle contains four domains with the aim to support patient transitions between outpatient and community/general practice settings. Other models of healthcare delivery do not overtly include culture or governance, domains necessary to enable effective communication, care coordination and overall high-quality service delivery. 44,56 The inner circle is composed of 6 operational areas (represented by coloured triangles). Each domain and operational area include criteria to provide guidance for the development of documents, systems and processes required within a nurse-led service. The domains, operational areas, criteria and supporting documents, systems and processes are further detailed in Table 3 .

\section{Operation of the model}

The patient is at the centre of the model and the key approach is for the nursing care coordinator to lead care coordination strategies that enable continuity of care for the patient between the outpatient setting and community/ general practice.

Continuity of care is important in healthcare delivery and good clinical outcomes. ${ }^{4,5}$ Coordination and continuity of care between hospital outpatient services and the community/general practice setting is part of the outpatient nurse's role and work. The nurse within this model will be the central point of contact for the patient and health team. The nurse's role is to facilitate optimisation of the patient's health status, liaise with medical and allied health professionals regarding complex health management, coordinate additional services, provide education or counselling, and support the patient's journey between the secondary and primary healthcare sectors. 
TABLE 3: DOMAINS, OPERATIONAL AREAS, CRITERIA AND SUPPORTING DOCUMENTS, SYSTEMS AND PROCESSES

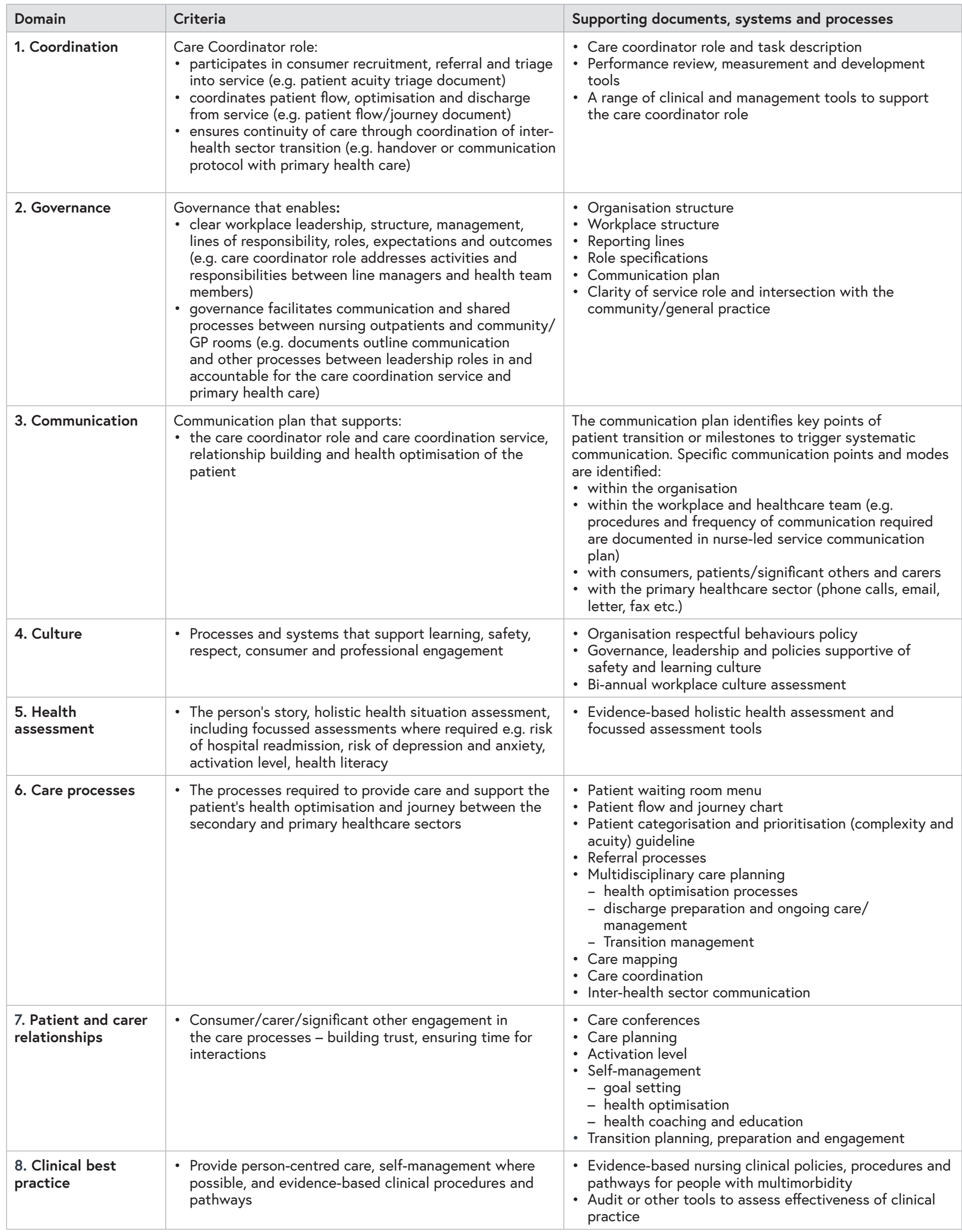


TABLE 3: DOMAINS, OPERATIONAL AREAS, CRITERIA AND SUPPORTING DOCUMENTS, SYSTEMS AND PROCESSES (continued)

\begin{tabular}{|c|c|c|}
\hline Domain & Criteria & Supporting documents, systems and processes \\
\hline $\begin{array}{l}\text { 9. Evaluation and } \\
\text { improvement }\end{array}$ & $\begin{array}{l}\text { - MACS team and person/consumer engaged with } \\
\text { evaluation and improvement, embedded quality } \\
\text { improvement in the service }\end{array}$ & $\begin{array}{l}\text { - Lead and contribute to quality improvement initiatives, } \\
\text { cycle and processes } \\
\text { - Lead and contribute to person/consumer engagement } \\
\text { with quality improvement cycle and processes } \\
\text { - Contribute to and implement nursing research within } \\
\text { MACS } \\
\text { - Contribute to continuing professional development } \\
\text { - } \text { education and up-skilling } \\
\text { - Suppl nursing key performance indicators } \\
\text { indicators }\end{array}$ \\
\hline $\begin{array}{l}\text { 10. Systems, } \\
\text { processes and } \\
\text { resources }\end{array}$ & $\begin{array}{l}\text { - Support safe skill mix, multidisciplinary work and } \\
\text { interactions/flexibility between nursing outpatients and } \\
\text { community/GP rooms }\end{array}$ & $\begin{array}{l}\text { - Support effective ongoing systems } \\
\text { - Lead, support and design new systems and processes: } \\
\text { apply } 10 \text { principles of 'good work design' (e.g. patient } \\
\text { flow and transition) }\end{array}$ \\
\hline
\end{tabular}

\section{DISCUSSION}

This nurse-led care coordination service model is evidencebased and relevant to the nursing best practice for management of people with multimorbidity. It incorporates key findings from the literature regarding best outcomes for people with multimorbidity, 8,24,32,57,58 and advances previous significant models of care (see Table 4). The Chronic Care Model and Transitional Care Model are two models that provide best practice management of people with chronic health conditions. ${ }^{27,32,59}$ Wagner's model takes a health systems approach, while Hirschman focuses on nurse-led multidisciplinary interventions that target chronically ill and complex adults or patients at risk for poor outcomes after discharge..$^{27,32}$ Additionally, the Australian Primary Healthcare Nurses Association has developed a series of building blocks to assist nurses in the primary healthcare sector to successfully initiate nurse-led services. ${ }^{.1}$ This model is designed to be implemented from the secondary healthcare sector, and through cross referencing with the above models, demonstrates the capacity to effectively intersect with the primary healthcare sector and advance care for people with multimorbidity.

The model places the patient at the centre of care, with the key approach of a nursing care coordinator to lead care coordination strategies that enable continuity of care for the patient between the outpatient setting (secondary care) and community/general practice (primary health care). Continuity of care is important in healthcare delivery and good clinical outcomes. ${ }^{4,5}$ Coordination and continuity of care between hospital outpatient services and the community/general practice setting is part of the outpatient nurse's role and work. The nurse within this model will be the central point of contact and 'go to' person for the patient and health team. The nurse's role is to facilitate optimisation of the patient's health status, coordinate additional services, provide education or counselling, and support the patient's journey between the secondary and primary healthcare sectors.
Each domain/component within the model is like the blood circulating from artery to arteriole and eventually cell. The intent being to provide a flow of information and guide the development and provision of more specific resources or systems for implementing the care coordination service (see Table 3); ensuring the right information is available at the right place. For example, within the coordination domain, a job and person specification document would be required to guide the effectiveness and outcomes of the care coordinator role. Similarly, as part of the care processes area, a waiting room menu was developed for patients to identify the top 3 issues they needed to address with the healthcare team during an appointment. ${ }^{60}$ Protocols were also developed for patient triage, acuity, and flow through the service, as well as nursing risk assessment and multidisciplinary care plans and other documents. A key goal of the nurse-led care coordination service is to return the patient to the primary healthcare sector for continued monitoring and management, reducing future avoidable hospitalisations. This new model of care is required because although it overlaps with other evidence-based models, the additional domains of governance and culture have not been overtly included in previous models. The additional domains could enable the best possibility for continuity of care between the secondary and primary healthcare sectors. Table 4 includes a comparison of the proposed model of care with previous models. It is acknowledged that not all aspects of each model are represented in the table below.

Collaborative development of the model with stakeholders such as healthcare consumers, secondary and primary health sector clinical practitioners, healthcare executives, academics and Aboriginal and the Torres Strait Island peoples, has resulted in a model that is both clinically applicable and evidence based. Pragmatism and adaptability were significant considerations when the model was developed, as these concepts prepare the model for use in the 'real world' clinical setting. Similarly, the domains of governance and culture enable the model to be pragmatic and adaptable, 
TABLE 4: MODELS OF CARE: CROSS-REFERENCING

\begin{tabular}{|c|c|c|c|c|}
\hline \multirow{2}{*}{$\begin{array}{l}\text { Current model (nurse-led } \\
\text { care coordination service) }\end{array}$} & \multicolumn{4}{|l|}{ Model characteristics/domains } \\
\hline & Wagner $^{27}$ & Mitchell2 ${ }^{2}$ & Hirschman 32 & APNA building blocks ${ }^{51}$ \\
\hline $\begin{array}{l}\text { Multidisciplinary inter health } \\
\text { sector collaboration }\end{array}$ & $\begin{array}{l}\text { Productive interactions, } \\
\text { prepared proactive practice } \\
\text { team }\end{array}$ & $\begin{array}{l}\text { Interdisciplinary } \\
\text { teamwork }\end{array}$ & $\begin{array}{l}\text { Fostering } \\
\text { coordination }\end{array}$ & Build the team \\
\hline 1. The person & Informed activated patient & & & Patient engagement \\
\hline 3. Governance & & & & Staffing and HR \\
\hline 4. Communication & $\begin{array}{l}\text { Productive interactions, } \\
\text { prepared proactive practice } \\
\text { team }\end{array}$ & $\begin{array}{l}\text { Communication and } \\
\text { information exchange }\end{array}$ & $\begin{array}{l}\text { Maintaining } \\
\text { relationships, } \\
\text { collaborating }\end{array}$ & \\
\hline \multicolumn{5}{|l|}{ 5. Culture } \\
\hline 6. Health assessment & & & $\begin{array}{l}\text { Assessing/managing } \\
\text { risks and symptoms } \\
\text { Screening }\end{array}$ & $\begin{array}{l}\text { Patient pathways, systems } \\
\text { and processes }\end{array}$ \\
\hline 7. Care processes & & $\begin{array}{l}\text { Access and } \\
\text { acceptability }\end{array}$ & $\begin{array}{l}\text { Educating/promoting } \\
\text { self-management }\end{array}$ & Systems and processes \\
\hline $\begin{array}{l}\text { 8. Patient and carer } \\
\text { relationships }\end{array}$ & & & $\begin{array}{l}\text { Engaging patients } \\
\text { and caregivers }\end{array}$ & Patient engagement \\
\hline 9. Clinical best practice & & $\begin{array}{l}\text { Use of shared } \\
\text { care guidelines or } \\
\text { pathways }\end{array}$ & & Best practice \\
\hline $\begin{array}{l}\text { 10. Evaluation and } \\
\text { improvement }\end{array}$ & & & & Evaluation and improvement \\
\hline $\begin{array}{l}\text { 11. Systems, processes and } \\
\text { resources }\end{array}$ & $\begin{array}{l}\text { Health Systems: } \\
\text { - Organisation of health care } \\
\text { - Delivery system } \\
\text { - Decision support } \\
\text { - Clinical information }\end{array}$ & $\begin{array}{l}\text { A viable funding } \\
\text { model, training and } \\
\text { education }\end{array}$ & Staffing & $\begin{array}{l}\text { A clear plan, systems and } \\
\text { processes, location, facilities } \\
\text { and funding } \\
\text { Staffing and HR }\end{array}$ \\
\hline
\end{tabular}

contributing to the potential for successful change management and model implementation in the clinical workplace. Developing a new model of care is significant but the challenge is to develop a model with valued potential to benefit patient care. In these times of disruption, it is acknowledged that consideration of change is required for successful introduction of a new model of care. ${ }^{61}$

The model developed from a pragmatic consultation process and literature review. It is anticipated that this pragmatic process will continue as implementation of care processes, documentation, systems, procedure development and nursing interventions associated with the domains and operational areas evolve. However, to ensure maximum uptake of the model, planning and preparation for its translation into practice is vital. ${ }^{62}$ Implementation will be challenging, ${ }^{63}$ and a collaborative process with stakeholders will be required. Identification of the culture within MACS and consultation with the MACS nurses and healthcare team, organisational leadership, primary healthcare nurses and practices and patients all associated with the MACS Unit will be necessary. The feasibility and effectiveness of the model will be assessed in subsequent action research cycles of the broader study. It is anticipated the model will evolve during the implementation of the nurse-led care coordination service. This will take place within the MACS clinic in the outpatient setting.

\section{LIMITATIONS}

This study was phase 1 in a pragmatic action research design, intended to engage a broad cross-section of stakeholders and provide clinicians with opportunities to participate and guide model development. However, there are limitations of the study that should be considered. The limitations included lack of a control or comparator group/model within the process, although previous models developed were considered and used as a standard to mitigate this. The setting in which team meetings and professional engagement and some validation took place was geographically limited - being present at one outpatient site only. This study employed a qualitative approach prohibiting validation and reliability testing of the stakeholder forum and validation workshop. This could be a consideration for future studies; incorporating a validated survey tool at workshops could augment data analysis and further validate the model. 


\section{IMPLICATIONS}

These findings provide a template and model for implementation of nurse-led services to guide clinical practitioners and nursing management from the secondary healthcare setting. The model's focus on person-centredness, coordination and communication augment the possibility of work between the secondary and primary healthcare sectors, providing a future pathway to enable effective health sector patient transition. Patient categorisation, pathways, and resource distribution, nursing roles and care guidelines have also been developed to support implementation of the model. Future systems, processes, education and work are required, but a foundation for change has been established. The collaborative and pragmatic approach to model development and supporting implementation resources, positions the model ideally for positive disruption and implementation rather than the risk of unplanned and unscheduled care delivery. ${ }^{47}$

Although an underlying barrier to effective transitions results from different funding sources for the secondary and primary healthcare sectors, this can be overcome by starting with small steps from local health networks, outpatient departments and primary healthcare practices. Nursing professional and industrial associations, as well as governments all recognise the need for new models of care and new nursing roles to address not only the complex care needs of people with multimorbidity, but also, increasing healthcare funding expenses. ${ }^{64,65}$ Changes in professional and structural boundaries are required to address the increasing complex needs of people with multimorbidity; this model provides direction for how this can be achieved.

\section{CONCLUSION}

A pragmatic approach drawing upon stakeholder consultation and evidence derived from relevant literature has been incorporated into the methodology of developing this nurse-led model of care. The model is person-centred, with all healthcare management activities intended to support and enable development of the person's agency in their healthcare optimisation. The model also focuses on nursing care coordination within a multidisciplinary approach and inter-health sector collaboration, to enable continuity of care between the secondary and primary healthcare sectors for people with multimorbidity. The model domains and criteria provide the potential for it to be adapted to nurse-led services providing care for a range of patients. Through stakeholder consultation across the health sector, consideration of the strengths of previous models and building blocks within the primary health sector, the model recognises patients' most pressing needs and provides a new pathway for transitional healthcare management. It includes an overarching component (multidisciplinary interhealth sector collaboration), four domains (co-ordination, governance, communication and culture) and six operational areas (health assessment, care processes, patient and carer relationships, clinical best practice, evaluation and improvement, and systems, processes and resources).

Part two in this series will report on the model feasibility and identify barriers and enablers to implementing a nurse-led care coordination service. The paper will report on patients', nurses' and healthcare staff experiences and identify the structures, processes and outcomes required to implement a nurse-led care coordination service. The feasibility of the nurse-led model to support continuity of care across the secondary and primary healthcare sectors for people with multimorbidity will be determined. The successful implementation of this model may provide a future pathway for implementation of nurse-led services both nationally and internationally.

Acknowledgements: The authors of the paper wish to thank all the stakeholders who contributed to the forums and validation workshop, particularly Kerri Reilly and Shereen Rankine who advised on terminology in reference to the representation of Aboriginal and Torres Strait Islander peoples', and the Central Adelaide Local Health Network (CALHN), particularly Multidisciplinary Ambulatory Consulting Service (MACS) staff. The authors also thank and acknowledge Allyson Rappensberg, Chronic Disease Nurse Consultant and Georgina Walker, Chronic Disease Clinical Nurse, who contributed to the development of the model for a nurse-led care coordination service.

Author contributions: KMD was responsible for the literature search, review and study design, drafting the initial manuscript and its revised versions. AH, MCE and GEC contributed significantly to the study design, drafting and preparation of the manuscript. JH, SS, and GS contributed to study design, drafting and preparation of manuscript.

Funding: This research received no specific grant from any funding agency in the public, commercial, or not-for-profit sectors.

Conflict of interest: No conflict of interest has been declared by the authors

\section{REFERENCES}

1 Davis KM, Eckert MC, Shakib S, et al. Development and Implementation of a Nurse-Led Model of Care Coordination to Provide Health-Sector Continuity of Care for People With Multimorbidity: Protocol for a Mixed Methods Study. JMIR Res Protoc. 2019; 8(12):e15006.

2 Mitchell GK, Burridge L, Zhang J, et al. Systematic review of integrated models of health care delivered at the primarysecondary interface: how effective is it and what determines effectiveness? Aust J Prim Health. 2015; 21(4):391-408.

3 Baxter S, Johnson M, Chambers D, Sutton A, Goyder E, Booth A. The effects of integrated care: a systematic review of UK and international evidence. BMC Health Serv Res. 2018; 18(1):350. 
4 Gjevjon ER, Romoren TI, Kjos BO, Helleso R. Continuity of care in home health-care practice: two management paradoxes. J Nurs Manag. 2013; 21(1):182-190.

5 Van Walraven C, Oake N, Jennings A, Forster AJ. The association between continuity of care and outcomes: a systematic and critical review. J Eval Clin Pract. 2010; 16(5) 947-956.

6 Haggerty JL, Reid RJ, Freeman GK, Starfield BH, Adair CE, \&, McKendry R. Continuity of care: A multidisciplinary review. BMJ (Online). 2003; 327(7425):3.

7 Reid RJ, Haggerty J, McKendry R. Defusing the confusion: concepts and measures of continuity of healthcare. Prepared for the Canadian Health Services Research Foundation; 2002.

8 Barnett K, Mercer SW, Norbury M, Watt G, Wyke S, Guthrie B. Epidemiology of multimorbidity and implications for health care, research, and medical education: a cross-sectional study. Lancet. 2012; 380(9836):37-43.

9 Bergenmar M, Nylén U, Lidbrink E, Bergh J, Brandberg Y Improvements in patient satisfaction at an outpatient clinic for patients with breast cancer. Acta Oncol. 2006; 45(5):550-558

10 Britt HC, Harrison CM, Miller GC, Knox SA. Prevalence and patterns of multimorbidity in Australia. Med J Aust. 2008; 189(2):72-77.

11 Glynn LG, Valderas JM, Healy P, et al. The prevalence of multimorbidity in primary care and its effect on health care utilization and cost. Fam Pract. 2011; 28(5):516-523.

12 Marengoni A, Angleman S, Melis R, et al. Aging with multimorbidity: a systematic review of the literature. Ageing Res Rev. 2011; 10(4):430-439.

13 Fortin M, Stewart M, Poitras M-E, Almirall J, Maddocks H. A systematic review of prevalence studies on multimorbidity: toward a more uniform methodology. Ann Fam Med. 2012; 10(2):142-151.

14 Le Reste JY, Nabbe P, Manceau B, et al. The European General Practice Research Network presents a comprehensive definition of multimorbidity in family medicine and long term care, following a systematic review of relevant literature. J Am Med Dir Assoc. 2013; 14(5):319-325.

15 Violan C, Foguet-Boreu Q, Flores-Mateo G, et al. Prevalence, determinants and patterns of multimorbidity in primary care: a systematic review of observational studies. PloS one. 2014. 9(7):e102149.

16 Afshar S, Roderick PJ, Kowal P, Dimitrov BD, Hill AG Multimorbidity and the inequalities of global ageing: a crosssectional study of 28 countries using the World Health Surveys. BMC Public Health. 2015; 15(1):776.

17 Pati S, Swain S, Hussain MA, et al. Prevalence and outcomes of multimorbidity in South Asia: a systematic review. BMJ open. 2015; 5(10):e007235

18 Garin N, Koyanagi A, Chatterji S, et al. Global Multimorbidity Patterns: A Cross-Sectional, Population-Based, Multi-Country Study. J Gerontol A Biol Sci Med Sci. 2016; 71(2):205-214.

19 Harrison C, Henderson J, Miller G, Britt H. The prevalence of complex multimorbidity in Australia. Aust N Z J Public Health. 2016; 40(3):239-244.

20 Mokraoui N-M, Haggerty J, Almirall J, Fortin M. Prevalence of self-reported multimorbidity in the general population and in primary care practices: a cross-sectional study. BMC Res Notes. 2016; 9(1):314
21 Hussain MA, Huxley RR, Al Mamun A. Multimorbidity prevalence and pattern in Indonesian adults: an exploratory study using national survey data. BMJ open. 2015; 5(12):e009810.

22 Sabaté E. Adherence to long-term therapies: evidence for action. Switzerland: World Health Organisation; 2003.

23 Wong FKY, Chow SKY, Chan TMF, Tam SKF. Comparison of effects between home visits with telephone calls and telephone calls only for transitional discharge support: a randomised controlled trial. Age and ageing. 2013; 43(1):91-97.

24 Smith SM, Soubhi H, Fortin M, Hudon C, O'Dowd T. Managing patients with multimorbidity: systematic review of interventions in primary care and community settings. BMJ. 2012; 345:e5205.

25 Pefoyo AJK, Bronskill SE, Gruneir A, et al. The increasing burden and complexity of multimorbidity. BMC Public Health. 2015; 15(1):415.

26 Splaine Wiggins $M$. The partnership care delivery model: An examination of the core concept and the need for a new model of care. J Nurs Manag. 2008; 16(5):629-638.

27 Wagner EH, Austin BT, Von MK. Improving outcomes in chronic illness. Manag Care Q. 1996; 4(2):12-25.

28 Kodner DL, Spreeuwenberg C. Integrated care: meaning, logic, applications, and implications-a discussion paper. Int J Integr Care. 2002; 2

29 Boult C, Karm L, Groves C. Improving chronic care: the "guided care" model. Perm J. 2008; 12(1):50.

30 Berra K. Does nurse case management improve implementation of guidelines for cardiovascular disease risk reduction? J Cardiovasc Nurs. 2011; 26(2):145-167.

31 Joo JY, Huber DL. An integrative review of nurse-led community-based case management effectiveness. Int Nurs Rev. 2014; 61(1):14-24.

32 Hirschman KB, Shaid E, McCauley K, Pauly MV, Naylor MD. Continuity of care: The transitional care model. Online J Issues Nurs. 2015; 20(3)

33 Brummel-Smith K, Butler D, Frieder M, et al. Person-Centered Care: A definition and essential elements. J Am Geriatr Soc. 2016; 64(1):15-18

34 Kitson A, Marshall A, Bassett K, Zeitz K. What are the core elements of patient-centred care? A narrative review and synthesis of the literature from health policy, medicine and nursing. J Adv Nurs. 2013; 69(1):4-15.

35 Corrigan JM. Crossing the quality chasm. Building a better delivery system. 2005.

36 Ekman I, Swedberg K, Taft C, et al. Person-centered careready for prime time. Eur J Cardiovasc Nurs. 2011; 10(4): 248-251.

37 McCormack B, McCance TV. Development of a framework for person-centred nursing. J Adv Nurs. 2006; 56(5):472-479.

38 Epstein RM, Street RL Jr. The values and value of patientcentered care. Ann Fam Med. 2011; 9(2):100-103.

39 Henbest RJ. Patient-centred care: a review of the concept. S Afr Fam Pract. 1989; 10(9).

40 Little P, Everitt H, Williamson I, et al. Preferences of patients for patient centred approach to consultation in primary care: observational study. BMJ Open. 2001; 322 (24).

41 Pelzang R. Time to learn: understanding patient-centred care Br J Nurs. 2010; 19(14) 
42 Robinson JH, Callister LC, Berry JA, Dearing KA. Patientcentered care and adherence: definitions and applications to improve outcomes. J Am Acad Nurse Pract. 2008; 20(12): 600-607.

43 Mannion R, Davies H. Understanding organisational culture for healthcare quality improvement. BMJ. 2018; 363:k4907.

44 Braithwaite J, Herkes J, Ludlow K, Testa L, Lamprell G. Association between organisational and workplace cultures, and patient outcomes: systematic review. BMJ Open. 2017; 7(11):e017708

45 Hastings SE, Armitage G, Mallinson S, Jackson K, E S. Exploring the relationship between governance mechanisms in healthcare and health workforce outcomes: a systematic review. BMC Health Serv Res. 2014; 14(479).

46 Nicholson C, Jackson C, Marley J. A governance model for integrated primary/secondary care for the health-reforming first world: results of a systematic review. BMC Health Serv Res. 2013; 13:528

47 Nam S. What's disruptive and what's not? Three criteria for identifying disruptors in healthcare. 2020. [Cited 2020 March 20] Available from: https://www.christenseninstitute.org/blog/ whats-disruptive-and-whats-not-three-criteria-for-identifyingdisruptors-in-healthcare/.

48 Government of New South wales. COVID-19 - Frequently asked questions. NSW Health. 2020. [cited 2020 March 20] Available from: https://www.health.nsw.gov.au/Infectious/alerts/Pages/ coronavirus-faqs.aspx\#1-2.

49 Bayliss EA, Edwards AE, Steiner JF, Main DS. Processes of care desired by elderly patients with multimorbidities. Fam Pract. 2008; 25(4):287-293.

50 Kemmis S, McTaggart R, Nixon R. The action research planner: doing critical participatory action research. In: Springer Science \& Business Media; 2014

51 APNA. Building blocks, explaining the essential elements of a nurse clinic. 2018. [cited 2018 February 13]. Available from: https://www.apna.asn.au/nursing-tools/nurse-clinics/ Buildingblocks.

52 Saunders M. Research methods for business students. New York: Pearson; 2015

53 Braun V, Clarke V. Using thematic analysis in psychology. Qual Res Psychol. 2006; 3(2):77-101.

54 Donabedian A. The quality of care: how can it be assessed? JAMA. 1988; 260(12):1743-1748.

55 World Health Organization. World Health Organization global strategy on people-centred and integrated health services: interim report. WHO. 2015.

56 Baker GR, Denis JL, Pomey MP, Maclntosh-Murray A. Designing effective governance for quality and safety in Canadian healthcare. Healthc Q. 2010; 13(1):38-45.

57 Caughey G. Ageing well ageing productively: people living with multiple chronic health conditions final report. South Australia: University of Adelaide, Flinders University, University of South Australia. 2013.

58 Bierman AS, Tinetti ME. Precision medicine to precision care: managing multimorbidity. Lancet. 2016; 388(10061):2721-2723.

59 Naylor MD, Sochalski JA. Scaling up: bringing the transitional care model into the mainstream. Issue Brief (Commonw Fund). 2010; 103:1-12.
60 Charlton J, Mackay L, McKnight JA. A pilot study comparing a type 1 nurse-led diabetes clinic with a conventional doctor-led diabetes clinic. European Diabetic Nurses Journal. 2004; 1(1):4

61 Corkin D, Kenny J. Quality patient care: challenges and opportunities. Nurs Manage (London). 2017; 24(7):32-36.

62 Grantham S, Knowles T, Nesin N, Truesdell N, Coakley E. Change is hard: what really happens when you try to implement a new care model. Fam Pract Manag. 2017; 24(6):10-15.

63 Molayaghobi NS, Abazari P, Taleghani F, et al. Overcoming challenges of implementing chronic care model in diabetes management: an action research approach. Int J Prev Med 2019; 10:13.

64 Struckmann V, Leijten FRM, Ginneken E, et al. Relevant models and elements of integrated care for multi-morbidity: results of a scoping review. Health Policy. 2018; 122:23-35.

65 Australian Institute of Health and Welfare. Australia's health 2018. In. Vol 16: AlHW; 2018 